\title{
Experimental and analytical evaluation of flow in a square-to-square wind tunnel contraction
}

\author{
Fuh-Min Fang*, J.C. Chen, Y.T. Hong \\ Department of Civil Engineering, National Chung-Hsing University, 250 Kuo-Kuang Road, \\ Taichung 40227, Taiwan
}

\begin{abstract}
Flow characteristics within a square-to-square contraction are investigated numerically and experimentally so as to gain additional insight into the contraction design. Measurements of cross-sectional velocity profiles and longitudinal pressure distributions along the wall centerlines are carried out in a contraction prototype and the results are compared to those predicted by the numerical simulations. Finally, the applicability of the nozzle design method, proposed by Fang (ASME J. Fluid Eng. (1997) 454), is assessed. Results show that although the inviscid calculation fails to simulate correctly the part of the nozzle flow near the contracting walls, the general behavior of the flow in the regions away from the wall is in reasonable agreement with the predicted behavior. On the other hand, the relative friction losses along the wall centerlines are about $4.3 \%$ relative to the total pressure drop through the contracting duct. After an adjustment is made in the normalization scheme, the measured pressure distributions are in good agreement with the numerically based distribution. (C) 2001 Elsevier Science Ltd. All rights reserved.
\end{abstract}

\section{Introduction}

A contraction is a major component of a wind or water tunnel. As the flow passes this transitional segment, it is accelerated so that a low-turbulence, uniform flow condition can be achieved in the test section. Generally, the contraction area ratio is the most dominant factor, which affects the extent of flow uniformity, the possibility of flow separation, and the downstream turbulence level. Once the contraction ratio

*Corresponding author. Tel.: + 886-4-2851707; fax: +886-4-2862857.

E-mail address: fmfang@dragon.nchu.edu.tw (F.-M. Fang). 
is determined, the nozzle shape and length, which also control the flow uniformity and the boundary layer growth along the contracting walls, become the next most important design requirements.

In principle, the problem of contraction design is a search for the optimum shape with minimum nozzle length for a desirable flow quality at the nozzle end. When the length is reduced, the contraction costs less and fits into a smaller space. In addition, the boundary layer will generally be thinner due to the combined effects of decreased length of boundary layer development and increased favorable pressure gradients in the contraction. However, the possibility of flow separation increases.

Typically, a contraction design starts with the selection of a contraction ratio (CR), which is mostly dictated by a consideration of the available space. Once the value of $\mathrm{CR}$ is determined, the nozzle shape and length must be chosen so as to satisfy predetermined design criteria, such as exit velocity profile non-uniformity, avoidance of separation, exit boundary layer thickness, space availability and cost.

By far, the most direct way for an engineering contraction design is that suggested by Morel [2,3]. He proposed the use of a combination of two matched cubics, each having its apex at one end of the contraction, as the basic shape of 2-D and axisymmetric contractions. When the dimensions of the upstream and downstream sections and the nozzle length are fixed, the location of the matched point becomes the only parameter to determine the entire wall shape. Morel's design procedure starts by prescribing a level of velocity non-uniformity at the contraction exit section, which leads to a corresponding minimum wall pressure near the exit. Based on design charts resulting from potential flow calculations, several possible contraction lengths can be selected corresponding to different locations of the matched point and 
to different maximum wall pressures near the inlet. After the Stratford criterion [4] is applied to check for the possibility of separation, the final choice among all the alternatives is the one which leads to the shortest length without the occurrence of separated flows.

For practical design reasons, Fang [1] extended Morel's procedure to a threedimensional square-to-square contraction case. With the three most important design factors selected as the avoidance of flow separation, an acceptable flow uniformity at the exit cross-section and minimizing the nozzle length, establishing a series of design charts were developed. Accordingly, a trial-and-error procedure is used to search for an optimum shape with minimum nozzle length for a prescribed flow quality.

\section{Present program}

With a prescribed contraction ratio of 9, a contraction prototype (Fig. 1) was built in an open-type wind tunnel system according to the design method proposed by Fang [1]. Velocity and pressure measurements were carried out and compared with numerical predictions. Results of the comparisons are used to evaluate the applicability of the design method.

The wind tunnel has a bell-mouth flare at the system entrance. The distances between the edges of the flare and the neighboring boundaries (ceiling, walls and ground) are 1.0, 0.85 and $1.0 \mathrm{~m}$, respectively. A 3-m-long settling chamber, containing a 10-cm-long honeycomb and three screens, follows the flare. Further downstream, the settling chamber is followed by the contraction and the test section.

Fig. 1 illustrates the geometry of the square-to-square nozzle prototype. The sizes of the inlet and exit cross-sections are, respectively, $2.40 \mathrm{~m} \times 2.40 \mathrm{~m}$ and $0.80 \mathrm{~m} \times$ $0.80 \mathrm{~m}$. The nozzle length $(L)$ is $3.60 \mathrm{~m}\left(L / H_{1}=1.50 ; H_{1}\right.$ is the width of the entrance section), and the location of the matched point $\left(x_{\mathrm{m}}\right)$ is selected as $1.80 \mathrm{~m}$ $\left(X_{\mathrm{m}}=x_{\mathrm{m}} / L=0.50\right)$. The shape of the contraction is the result of a combination

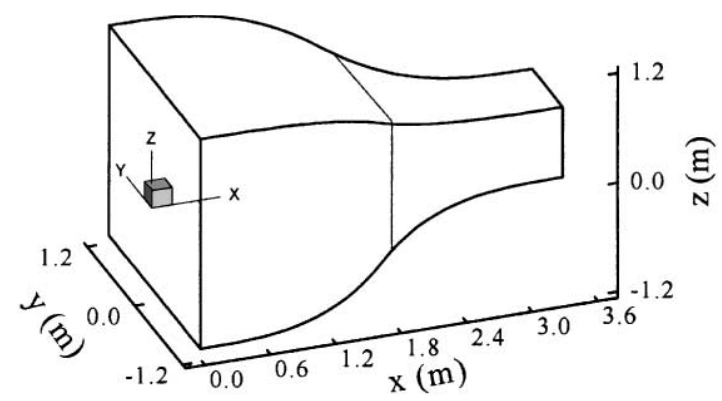

Fig. 1. Description of the contraction prototype. 


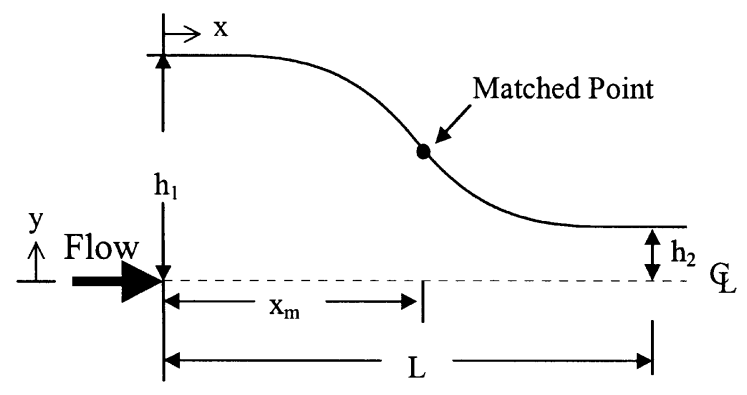

Fig. 2. Schematic of the contraction (half plane).

of two matched cubics, described as (also see Fig. 2)

$$
\begin{gathered}
y(\text { or } z)=\left(h_{1}-h_{2}\right)\left[1-\frac{1}{X_{\mathrm{m}}^{2}}\left(\frac{x}{L}\right)^{3}\right]+h_{2}, \quad x<x_{\mathrm{m}}, \\
\text { or }=\frac{\left(h_{1}-h_{2}\right)}{\left(1-X_{\mathrm{m}}\right)^{2}}\left(1-\frac{x}{L}\right)^{3}+h_{2}, \quad x>x_{\mathrm{m}},
\end{gathered}
$$

where $h_{1}\left(=0.5 H_{1}\right)$ and $h_{2}$ are, respectively, the half-widths of the inlet and exit sections of the nozzle.

\subsection{Numerical method}

The numerical computations adopt a finite-volume Euler solver [5] to obtain inviscid flow results within the contraction. Under the assumption that the flow density is a function only of pressure (barotropic), for a low-Mach-number flow, the equations of motion can be approximated in a conservative form as

$$
\frac{\partial G_{\mathrm{i}}}{\partial t}+\nabla \cdot \boldsymbol{F}_{i}=0, \quad i=1,2,3,4
$$

where

$$
\boldsymbol{G}=\left[\begin{array}{c}
p \\
u \\
v \\
w
\end{array}\right] ; \quad \boldsymbol{F}=\left[\begin{array}{ccc}
K u & K v & K w \\
u^{2}+p / \rho & u v & u w \\
u v & v^{2}+p / \rho & v w \\
u w & v w & w^{2}+p / \rho
\end{array}\right]
$$

$\rho$ is the density; $p, u, v$ and $w$ are pressure and velocity components, and $K$ is the bulk modulus of elasticity.

Computation proceeds by using a volume integration over a specific control volume $(\forall)$. Based on the divergence theorem, one has

$$
\frac{\partial G_{\mathrm{m}}}{\partial t}=-\frac{1}{\forall} \int_{\forall} \boldsymbol{n} \cdot \boldsymbol{F} \mathrm{d} S,
$$


where $G_{\mathrm{m}}$ represents the mean quantity with reference to the center of the volume; $\boldsymbol{n}$ is the normal vector of the control surface. This equation is used to calculate the change of $G_{\mathrm{m}}$ at a starting time step within an elapsed period $(\Delta t)$ and thus update the $G_{\mathrm{m}}$ values for the next time step.

In the simulations, appropriate values of pressures and velocities are specified at exterior (phantom) cells outside the boundaries to reflect the correct physical nature of the local boundaries. For the contracting walls, a slip condition is used for the velocity specifications, and the pressures at the phantom cells are given by taking into account the effect of the wall curvature. At the inlet and exit boundaries of the nozzle, on the other hand, the phantom-cell velocities are specified according to the fact that the total head is constant. Finally, the pressures at the entrance are given based on a zero-gradient assumption, and the average pressure at the exit crosssection is taken as the reference pressure of the flow in the computational domain.

\subsection{Experimental set-up}

Three rows of pressure taps are set along the centerlines of the top, bottom and one of the side walls of the contraction prototype. All taps are connected to a micromanometer with a resolution of $\frac{1}{1000}$ of a centimeter. These allow for accurate evaluations of the longitudinal pressure distributions.

In addition, hot-wire anemometry is used to measure the mean velocity ( $x$ component) profiles at four selected cross-sections within the nozzle $(x / L=$ $0.32,0.51,0.68$ and 1.0). For a typical velocity measurement, signals are recorded within a period of $2 \mathrm{~min}$ with a sampling rate of 2 samples/s. At each cross-section, the velocity is measured at a distance varying from 2 to $8 \mathrm{~cm}$ and a total of 144 velocity measurements are conducted.

\section{Results}

In the study, the measurements and numerical simulations are performed under the condition that the velocity at the nozzle exit section is $15 \mathrm{~m} / \mathrm{s}\left(u_{\mathrm{e}}\right)$. The corresponding Reynolds number, in terms of the velocity and the width of the exit section, is about $8 \times 10^{5}$.

\subsection{Mean velocity profiles at various cross-sections}

Based on the results of the inviscid calculations, Fig. 3a shows the normalized mean velocity profiles at the selected cross-sections $\left(H_{x}\right.$ being the local width of the section). Since the flow domain is considered symmetric to the centerline of the nozzle in the computations, the patterns of the calculated results also appear symmetric. At the three upstream sections $(x / L<0.68)$, higher speeds are found at the sectional center regions. At the contraction exit $(x / L=1.0)$, the trend of the cross-sectional velocity variation becomes the opposite. That is, without considering 

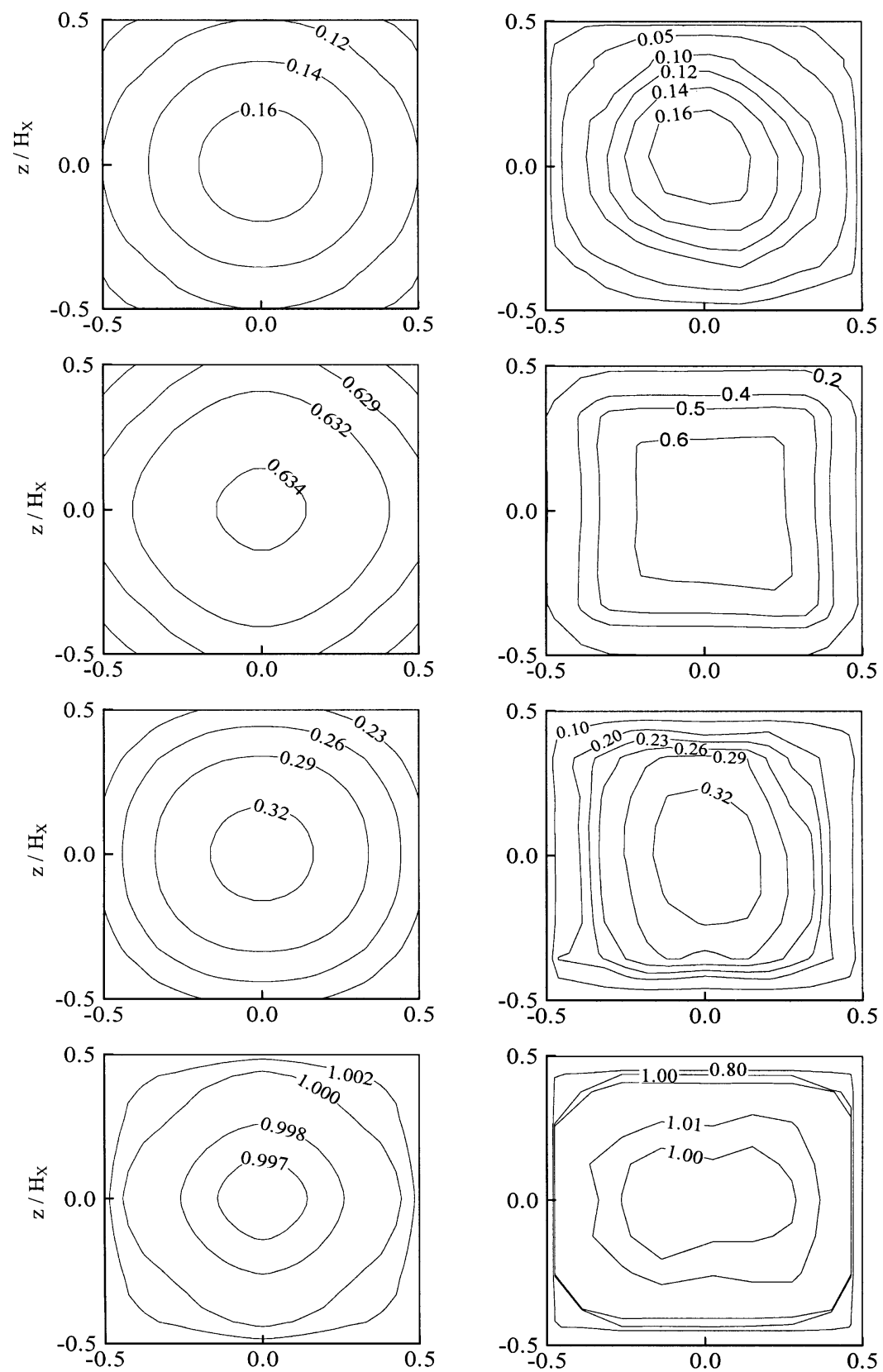

(a)

$\mathrm{y} / \mathrm{H}_{\mathrm{x}}$

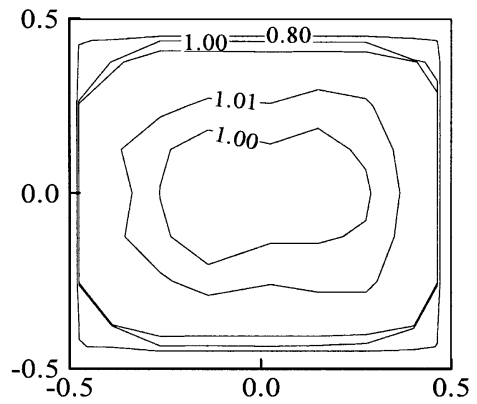

(b)

$$
\mathrm{y} / \mathrm{H}_{\mathrm{X}}
$$

Fig. 3. Sectional velocity profiles $(x / L=0.32,0.51,0.68$ and 1.0$)$ : (a) calculated; (b) measured. 


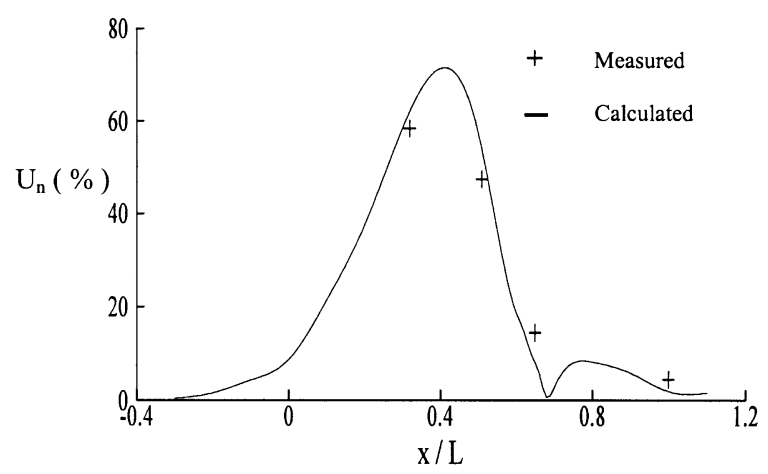

Fig. 4. Variations of velocity non-uniformity.

the region near the wall, larger speeds occur near the corners, while smaller speeds occur at the center regions.

Compared to the calculated results, Fig. $3 \mathrm{~b}$ depicts the corresponding mean velocity profiles from the experiments. In all cases, large transverse velocity gradients, which the inviscid computations fail to predict, are obtained in the nearwall regions due to the existence of boundary layers in the real flow. In the regions away from the wall, on the other hand, the measurement results appear to agree reasonably well with those from the calculations.

\subsection{Variations of the cross-sectional velocity non-uniformity}

Fig. 4 depicts the calculated cross-sectional velocity non-uniformity $\left(U_{\mathrm{n}}\right)$ variation, where $U_{\mathrm{n}}$ represents the ratio (in percentage) of maximum velocity deviation to the local cross-sectional mean velocity. In the simulated flow, the non-uniformity starts with a small value then increases as it goes downstream. As $x / L$ reaches about $0.4, U_{\mathrm{n}}$ becomes maximum. After that, a minimum value of $U_{\mathrm{n}}$ is obtained as $x / L$ equals about 0.70 . Further downstream, the $U_{\mathrm{n}}$ value again increases then decreases.

In comparison, the experimental data, obtained without the consideration of the near-wall regions, agree well with the result of the calculation (also see Fig. 4).

\subsection{Longitudinal pressure distributions}

By taking the pressure at the most upstream tap as the reference pressure, Fig. 5 shows the measured pressure variations along the centerlines of the top, side and bottom walls. In contrast to the corresponding calculated results, the estimated friction losses along the streamlines are about $5.8 \mathrm{~N} / \mathrm{m}^{2}$, corresponding to $0.59 \mathrm{~mm}$ of water head. 


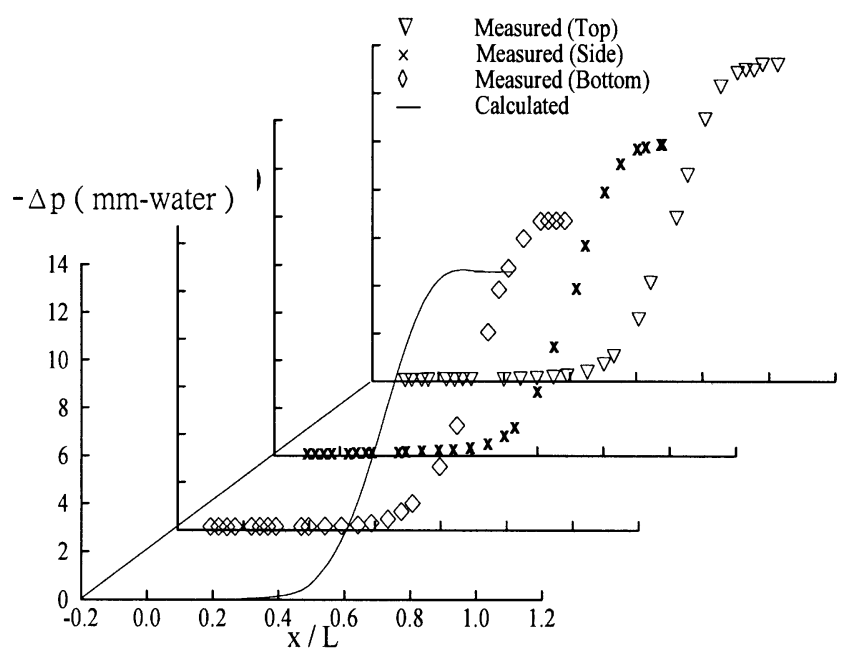

Fig. 5. Comparison of pressure distribution along wall centerlines.

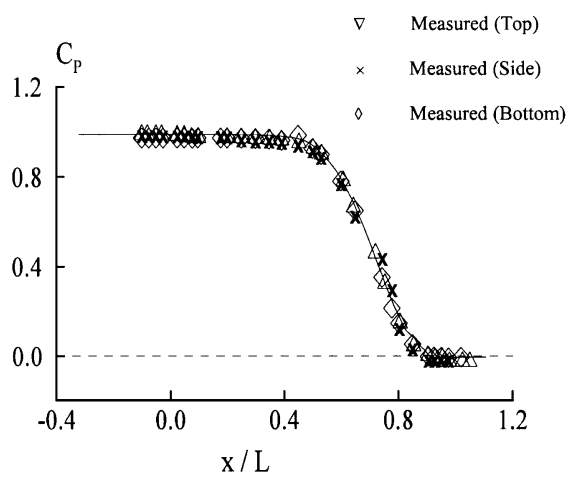

Fig. 6. Normalized pressure distributions along wall centerlines.

To normalize both the measured and calculated results in a more consistent manner, an adjusted dimensionless parameter is used as

$$
C_{p}=\frac{p-p_{\mathrm{e}}}{0.5 \rho u_{\mathrm{e}}^{2}} \frac{\left(p_{\mathrm{i}}-p_{\mathrm{e}}\right)_{\mathrm{cal}}}{\left(p_{\mathrm{i}}-p_{\mathrm{e}}\right)_{\text {meas }}}
$$

where $p_{\mathrm{i}}$ and $p_{\mathrm{e}}$ are referred respectively to those along the centerlines at the inlet and exit sections of the contraction; the subscripts "cal" and "meas" stand for the calculated and measured values. Accordingly, all the normalized pressure distributions become almost identical, as shown in Fig. 6. 


\section{Discussion}

\subsection{Flow predictions and measurements}

From the viewpoint of nozzle designs, the application of numerical methods in flow analyses always results in errors as the computational domain is truncated into a simplified one. In the simulation, the selected domain of computations contains the portion of the nozzle, preceded and followed by a straight duct with a length of onehalf the width of the contraction end section $\left(h_{1}\right.$ and $\left.h_{2}\right)$. Further numerical investigations reveal that the relative error produced by this selection is not more than $3 \%$.

The assumption in the computation is that in the absence of separation, the turbulence intensity of the flow should be rather low due to the effect of large acceleration in the contraction. For the same reason, moreover, the boundary layer thickness along the contracting walls is generally thin in comparison with the width of the local cross-section. Therefore, the non-separated, low-turbulence flow outside the boundary layers can be considered as an inviscid one with acceptable accuracy, except at the regions near the walls [1]. The results of the experimental investigations show that the boundary layer thickness along the wall centerlines varies from a few centimeters to about $1 \mathrm{~cm}$ in the nozzle prototype, indicating that the error introduced by the use of the inviscid assumption becomes less significant as it goes downstream.

Moreover, although a honeycomb and screens are set upstream the contraction prototype in the wind tunnel system to precondition the inlet flow, the actual flow condition at the nozzle inlet section may still be somewhat different from that used in the numerical calculation. This could explain why the actual velocity profiles were not accurately predicted by the numerical methods (Fig. 3). Unfortunately, due to practical limitations, the velocity profile at the nozzle inlet could not be measured to support the above statement.

In the experiments, another difficulty encountered is that the application of hotwire anemometry does not provide enough accuracy to resolve mild velocity variations, especially at the exit section, where the velocity deviates only a few percent. A more appropriate technique or method is therefore needed to solve this problem.

\subsection{Behavior of mean flow in the contraction}

When a flow passes a nozzle, the increase in mean speed can smooth out spatial irregularities contained in the flow. For any finite-length contraction, there exist two adverse pressure gradients, where the flow might possibly separate [2]. As the contraction is designed in a way that flow separation is avoided, the majority of the flow should be well described by inviscid analyses [1]. Further examinations on the cross-sectional velocity profiles reveal that the nozzle flow is free of separation, which is the expectation of the original design. 
It is generally known that due to the effects of wall curvature, the velocity profile at any section within the nozzle is always non-uniform. The numerical results in Fig. 3a show that higher velocities occur in the core region at the three upstream measured cross-sections. On the other hand, the trend of the sectional velocity variations becomes the opposite at the nozzle exit. Despite the inconsistency between the predicted and measured velocity profiles in Fig. 3, a similar trend is found from the results of measurements (Fig. 3b).

The streamwise variation of the cross-sectional velocity non-uniformity shown in Fig. 4 is also interesting. Although the inlet velocity profile (at $x / L=0$ ) is considered fairly uniform $\left(U_{\mathrm{n}}\right.$ is about $5 \%$ ), the numerical prediction shows that the value of velocity non-uniformity increases significantly at the beginning then reaches a peak value of about $71 \%$ as $x / L$ is equal to 0.41 . Further downstream, the value of $U_{\mathrm{n}}$ decays sharply until $x / L$ equals 0.70 . The result in Fig. 4 indicates that without the consideration of the near-wall regions the experimental data of $U_{\mathrm{n}}$ are consistent with the predicted variations.

\subsection{Patterns of cross-sectional velocity profiles}

Further simulations were conducted to investigate the effect of the contraction ratio $(\mathrm{CR}=4,9,12$ and 16$)$ on the cross-sectional flow patterns with the same nozzle length $\left(L=1.5 H_{1}\right)$ and the location of the matched point $\left(X_{\mathrm{m}}=0.5\right)$, Based on the numerical results, Fig. 7 illustrates the normalized velocity profiles at four selected cross-sections $(x / L=0.2,0.4,0.6$ and 0.8$)$ for different $C R$ values. It can be seen in Fig. 8 that the patterns of the cross-sectional non-uniformity $\left(U_{\mathrm{n}}\right)$ variations appear rather similar in all cases. At the beginning of the contraction, the value of $U_{\mathrm{n}}$ increases significantly then reaches a peak value. Fig. 9 shows that the location of this peak value appears insensitive to the CR value $(x / L$ is about 0.4$)$. However, an increase of CR leads to an increase of $U_{\mathrm{n}}$. Further downstream, $U_{\mathrm{n}}$ decays sharply and reaches a minimum value. The location of the cross-section, corresponding to the minimum $U_{\mathrm{n}}$ value, increases (see Fig. 10) as CR increases. Further examinations on the velocity profiles before and after this cross-section reveal that this location corresponds to a transition of the transverse deviation pattern of the cross-sectional velocity profile (Fig. 11). In all cases, upstream of this cross-section, higher speeds are found at the sectional center region. Downstream of this cross-section, on the other hand, the trend of the transverse deviation of the cross-sectional velocity profile becomes the opposite.

Finally, although a larger CR value results in a larger peak value of $U_{\mathrm{n}}$ in the middle section (at $x / L$ equals about 0.4 ) within the contraction (Figs. 8 and 9), the cross-sectional velocity profile becomes more uniform as CR increases (Fig. 12). This result agrees with common contraction design principles.

\subsection{Longitudunal pressure distributions along the wall centerlines in the contraction}

By examining the measured pressure distributions along the wall centerlines (Fig. 5), it is found that the difference is insignificant, implying that the flow 

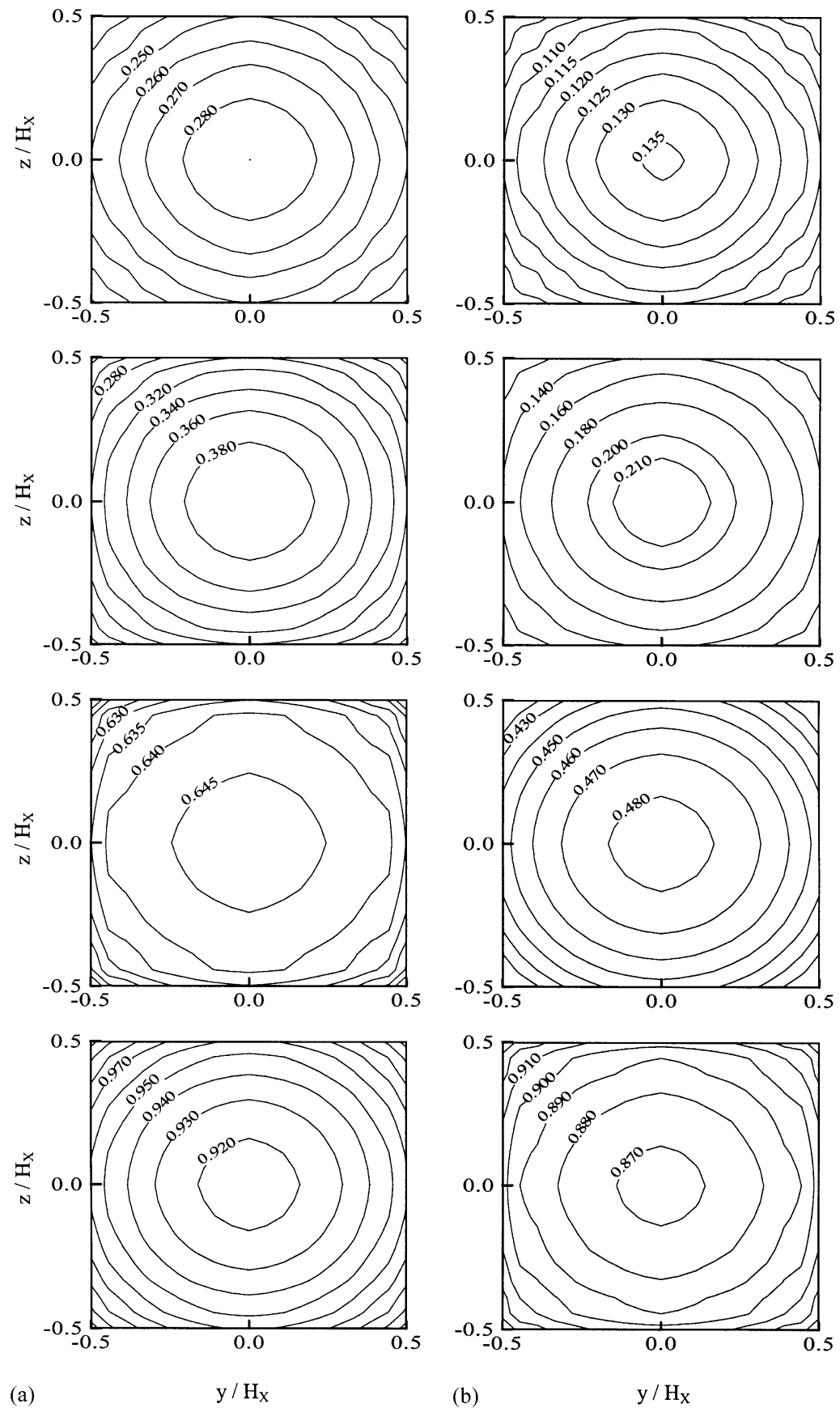

Fig. 7. Normalized cross-sectional velocity profiles $(x / L=0.2,0.4,0.6$ and 0,8$)$ : (a) $\mathrm{CR}=4$; (b) $\mathrm{CR}=9$; (c) $\mathrm{CR}=12$; (d) $\mathrm{CR}=16$. 

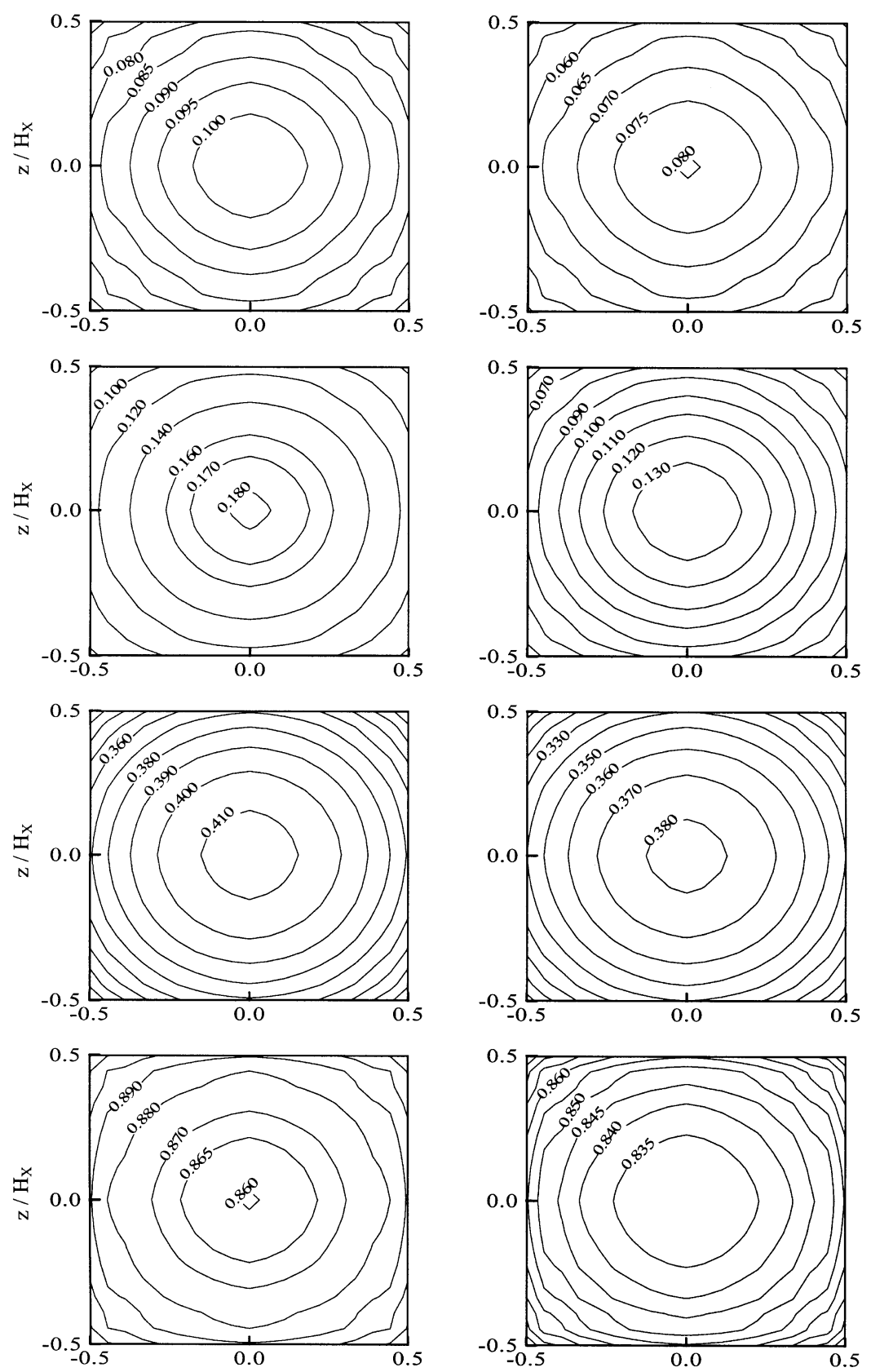

(c)

$\mathrm{y} / \mathrm{H}_{\mathrm{X}}$

(d)

$\mathrm{y} / \mathrm{H}_{\mathrm{X}}$

Fig. 7. Continued. 


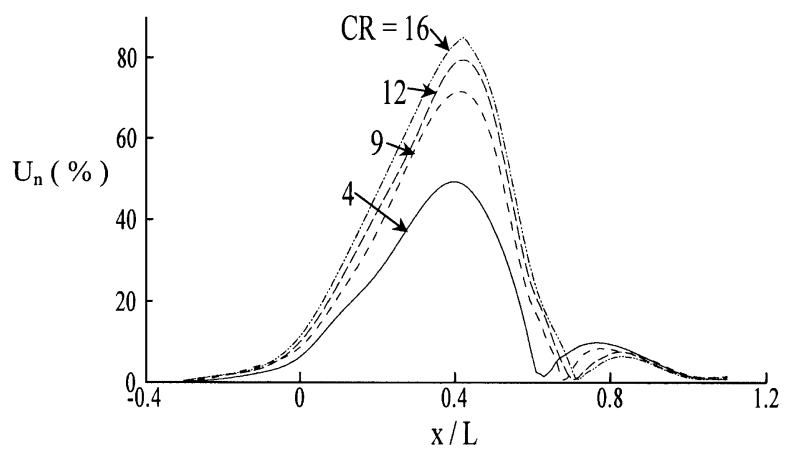

Fig. 8. Variations of velocity non-uniformity for different CR values.

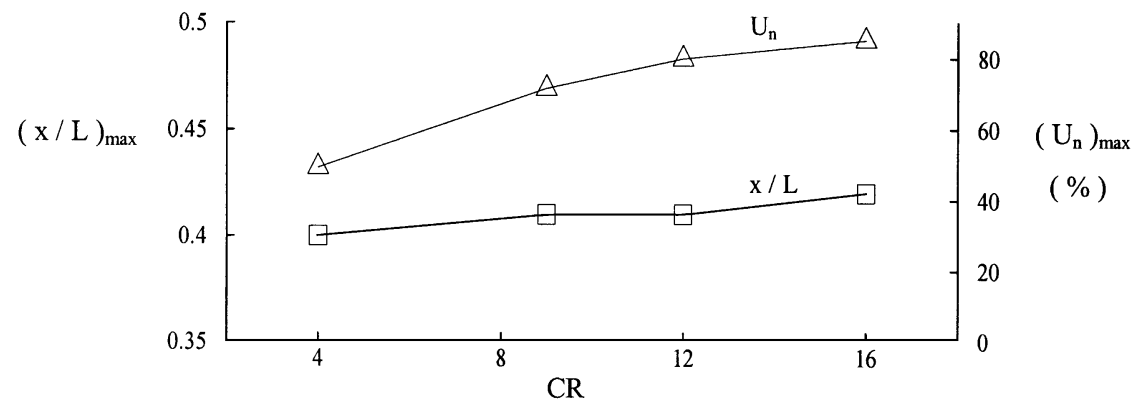

Fig. 9. Maximum $U_{\mathrm{n}}$ values and the locations for different $\mathrm{CR}$ values.

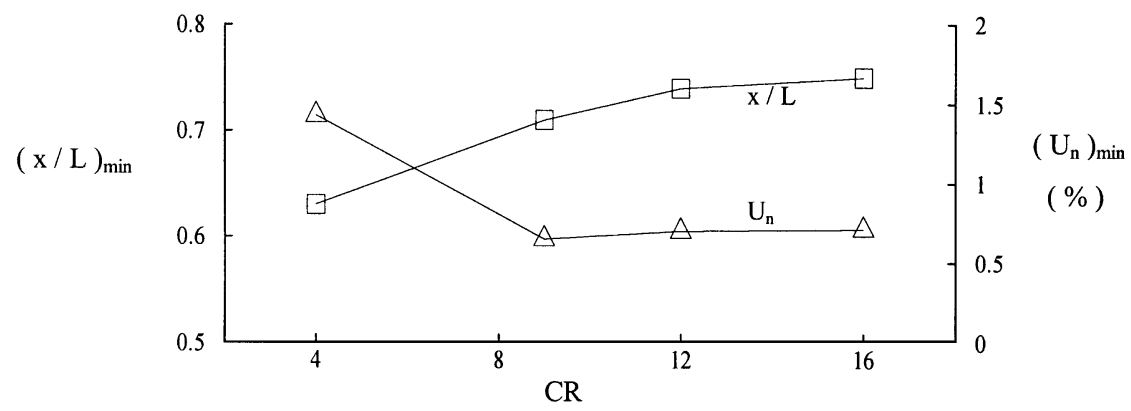

Fig. 10. Minimum $U_{\mathrm{n}}$ values and the locations for different $\mathrm{CR}$ values.

behaviors near the three centerlines are rather similar. The symmetry of crosssectional velocity profiles can also be detected from the results in Fig. $3 \mathrm{~b}$.

Due to the existence of wall friction, the actual Bernoulli sum along the streamlines decreases. If the wall centerlines can be treated as streamlines of the mean flow, experimental results of the pressure distributions show that the head 


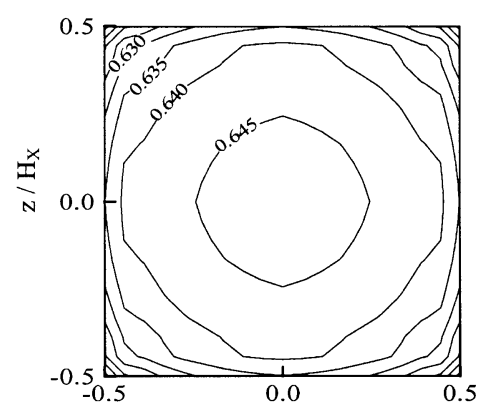

$\mathrm{CR}=4$

(a) $\mathrm{x} / \mathrm{L}=0.62$

(b) $\mathrm{x} / \mathrm{L}=0.63$
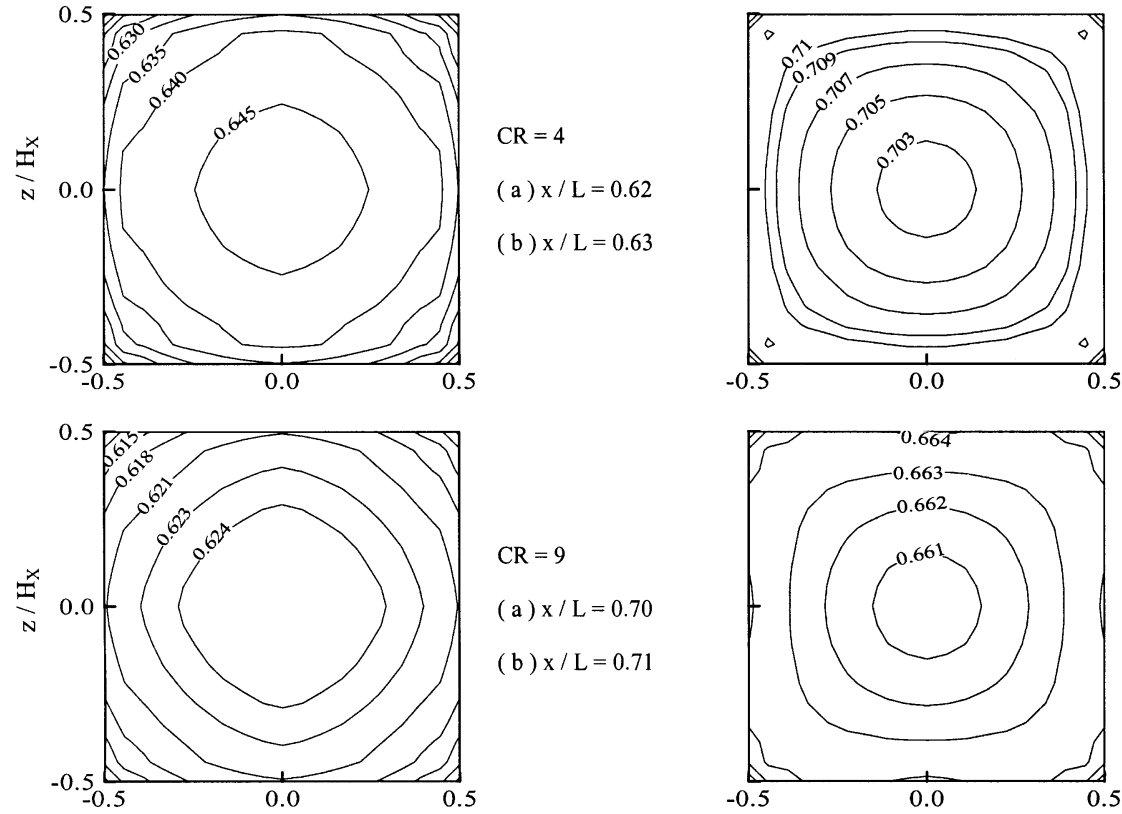

$\mathrm{CR}=9$

(a) $\mathrm{x} / \mathrm{L}=0.70$

(b) $\mathrm{x} / \mathrm{L}=0.71$
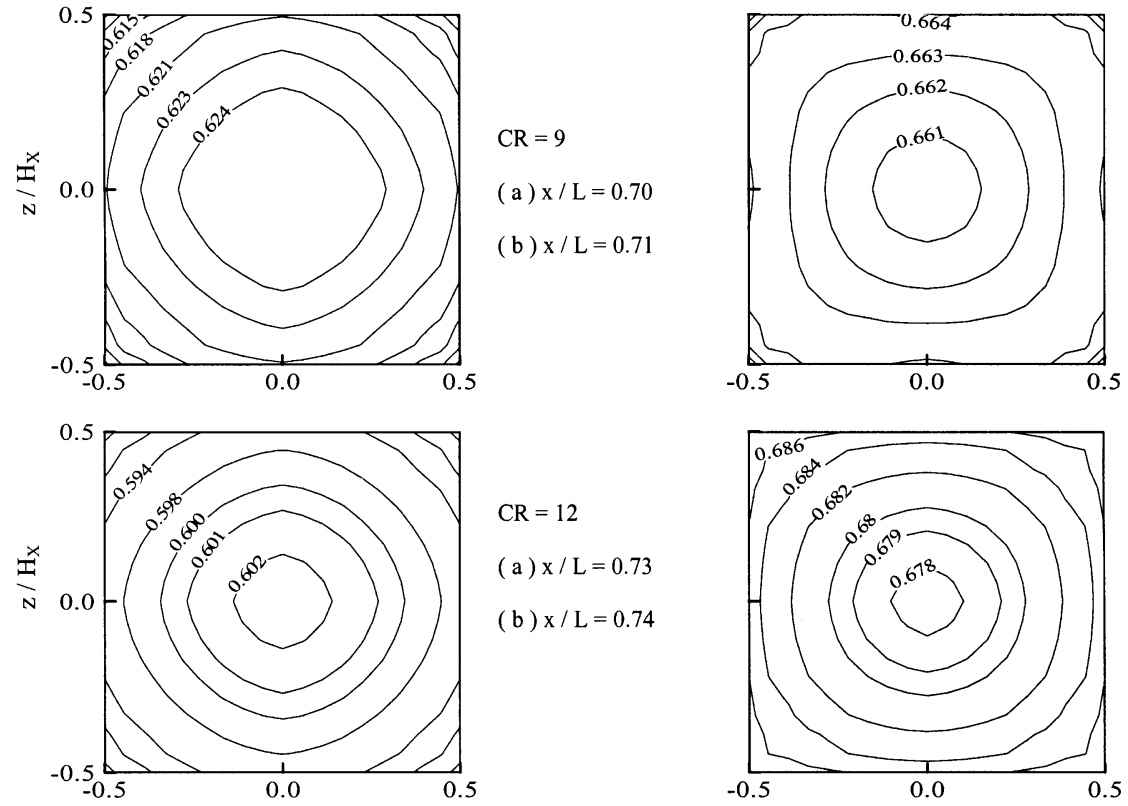

$\mathrm{CR}=12$

(a) $\mathrm{x} / \mathrm{L}=0.73$

(b) $\mathrm{x} / \mathrm{L}=0.74$
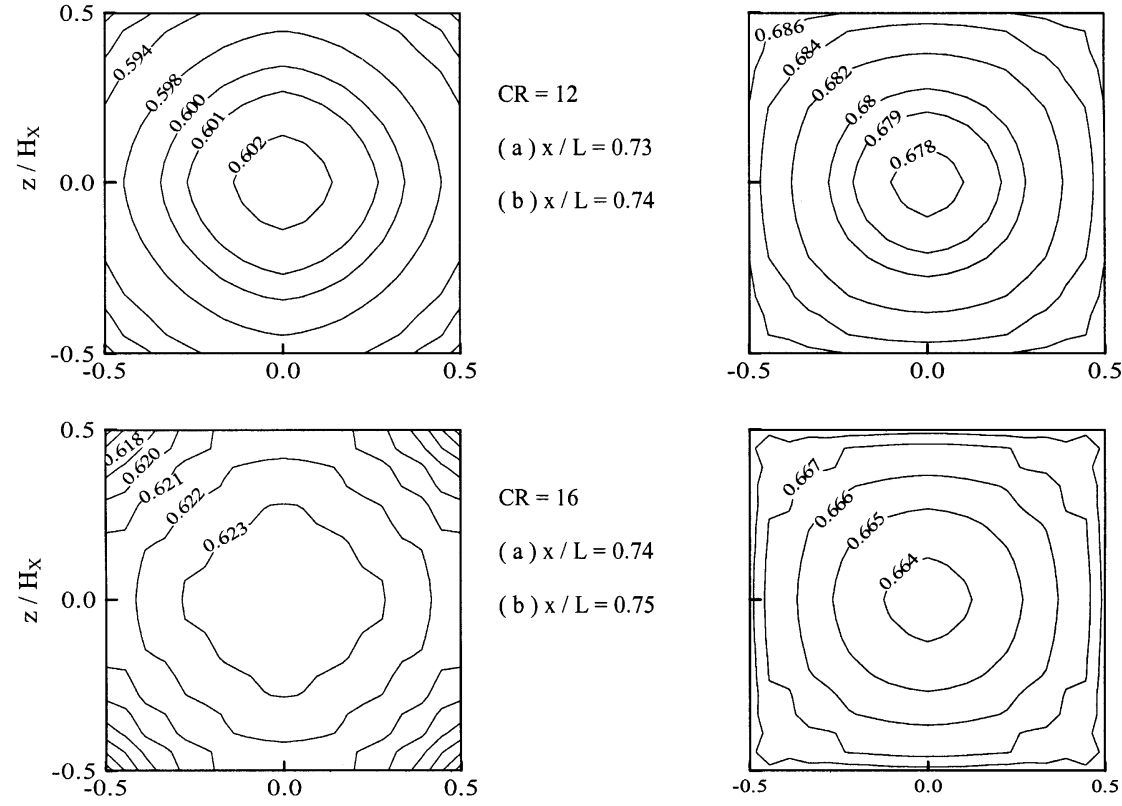

(a) $\mathrm{y} / \mathrm{H}_{\mathrm{X}}$

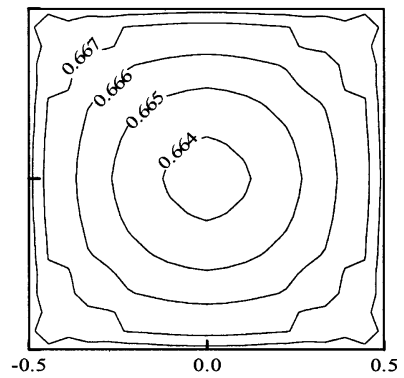

(b)

$\mathrm{y} / \mathrm{H}_{\mathrm{X}}$

Fig. 11. Normalized velocity profiles before and after the transitional cross-sections. 


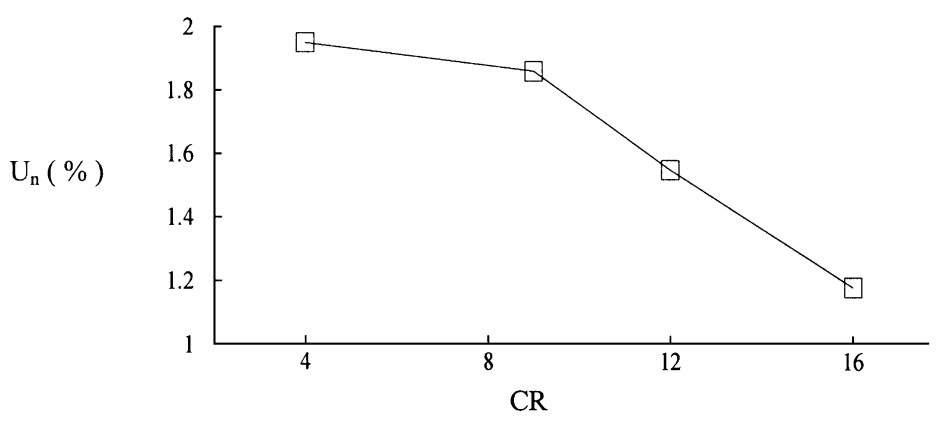

Fig. 12. Velocity non-uniformity at the nozzle end cross-section.

losses in all cases are about $5.8 \mathrm{~N} / \mathrm{m}^{2}$, approximately $4.3 \%$ relative to the total pressure drop through the contracting duct. For a better comparison between the numerical and experimental results of the centerline pressure distributions, the selection of the normalized pressure $\left(C_{p}\right)$, defined by Eq. (5), is considered adequate to interpret both sets of data in a more consistent manner. By using this parameter, Fig. 6 shows that the measurement results are in good agreement with the numerical one.

\subsection{Comments on the design method}

The design of a wind tunnel system involves a tremendous amount of work due to its complexity. At the stage of the preliminary design, since it is practically not possible to analyze the flow in the entire system, it is generally convenient to divide the system into several segments so as to proceed with flow analyses and the design of each individual component. The basic concept of the contraction design method [1] as well as that in the present numerical study adopts this approach.

Compared to the results of flow measurements in the nozzle, it has been found that the numerical simulation suffers from certain uncertainties, such as the inconsistency of the actual and numerical flow conditions at the nozzle inlet. Qualitatively, however, the overall flow behavior in the nozzle appears well predicted except in the near-wall region. On the other hand, if the friction loss of the contraction can be accurately estimated, the pressure distributions along the contracting wall can then be achieved based on the numerical results. This would allow for a more extensive check on the possibility of the occurrence of flow separation.

\section{Conclusion}

Flow characteristics, within a square-to-square contraction, have been investigated numerically and experimentally so as to gain further insight into the contraction design. Additionally, by comparing the measurement and the corresponding 
numerical results of flow behavior in a contraction prototype, the applicability of the nozzle design method proposed by Fang [1] has been assessed.

\section{Acknowledgements}

The study was funded by a Grant (NSC 88-2611-E-005-005) from the National Science Council. The computing time was provided by the National Center for High-Performance Computing in Taiwan.

\section{References}

[1] F. Fang, A design method for contractions with square end sections, ASME J. Fluids Eng. 119 (1997) 454- 458.

[2] T. Morel, Comprehensive design of axisymmetric wind tunnel contractions, ASME J. Fluids Eng. 97 (1975) 225-233.

[3] T. Morel, Design of two-dimensional wind tunnel contractions, ASME J. Fluids Eng. 99 (1977) 371-378.

[4] B.S. Stratford, The prediction of separation of the turbulent boundary layer, J. Fluid Mech. 5 (1959) $1-16$.

[5] C.C.S. Song, M. Yuan, A weakly compressible flow model and rapid convergence methods, ASME J. Fluids Eng. 110 (1988) 441-455. 